\title{
On MAP Symbol Detection for ISI Channels Using the Ungerboeck Observation Model
}

\author{
Giulio Colavolpe and Alan Barbieri
}

\begin{abstract}
In this letter, the well-known problem of a transmission over an additive white Gaussian noise channel affected by known intersymbol interference is considered. We show that the maximum a posteriori (MAP) symbol detection strategy, usually implemented by using the Forney observation model, can be equivalently implemented based on the samples at the output of a filter matched to the received pulse, i.e., based on the Ungerboeck observation model. Although interesting from a conceptual viewpoint, the derived algorithm has a practical relevance in turbo equalization schemes for partial response signalling, where the implementation of a whitening filter can be avoided.
\end{abstract}

Index Terms-Factor graphs (FG), intersymbol interference (ISI), sum-product algorithm (SPA).

\section{INTRODUCTION}

W HEN the maximum a posteriori (MAP) sequence detection strategy is considered for transmissions over known intersymbol interference (ISI) channels, two equivalent approaches for linear modulations can be adopted. The first one is the so-called Ungerboeck approach [1]. In this case, the branch metrics of the Viterbi algorithm (VA) implementing this strategy are based on the samples at the output of a filter matched to the received pulse. The second approach is the Forney approach [2] and it is based on the output of a whitened matched filter (WMF). Although the relevant VA branch metrics are different, the number of trellis states is the same for both equivalent approaches.

When the MAP symbol detection strategy is adopted, as an example to perform turbo equalization [3]-[5], these two observation models do not seem to be equivalent. All the wellknown materialization of the MAP symbol detection strategy, such as for example the BCJR algorithm [6] (see also [7], [8]) have been obtained by using a probabilistic derivation based on the chain rule and the properties of a Markov source observed through a discrete memoryless channel. Hence, this derivation cannot be directly extended to the case of the Ungerboeck observation model. In this letter, we solve this problem by using a properly defined factor graph (FG) and the sum-product algorithm (SPA) [9].

\section{PRELIMINARIES}

In this section, we briefly describe the FGs and the SPA, and summarize the results on MAP sequence detection for ISI channels.

Manuscript received December 14, 2004. The associate editor coordinating the review of this letter and approving it for publication was Prof. Jing Li. This paper was presented in part at the IEEE Symposium on Information Theory and its Applications (ISITA'04), Parma, Italy, October 2004.

The authors are with the Università di Parma, Dipartimento di Ingegneria dell'Informazione, Parma, Italy (e-mail: giulio@unipr.it, barbieri@tlc.unipr.it).

Digital Object Identifier 10.1109/LCOMM.2005.08003.

\section{A. Factor Graphs and the Sum-Product Algorithm}

A FG is a bipartite graph which expresses the way a complicated joint probability mass function (pmf) or a joint probability density function (pdf) of many variables factors into the product of local functions (not necessarily pmfs or pdfs) [9]. Let $V=\left\{v_{1}, \ldots, v_{N}\right\}$ denote a set of variables and $F(V)$ a multivariate function. Let $V_{1}, \ldots, V_{m}$ denote subsets of $V$. We say that $F(V)$ admits a factorization with supports $V_{1}, \ldots, V_{m}$, if $F(V)$ can be written as the product of the functions $\left\{F_{j}: j=1, \ldots, m\right\}$, where $F_{j}$ has the variables in $V_{j}$ as arguments. The $\mathrm{FG}$ representing the factorization $F=\prod_{j} F_{j}$ is a bipartite graph $\mathcal{G}=\{\mathcal{V}, \mathcal{F}, \mathcal{E}\}$, where nodes in $\mathcal{V}$ (variable nodes) are associated with the variables $v_{i} \in V$, nodes in $\mathcal{F}$ (factor nodes) are associated with the functions $F_{j}$, and there exists an edge $e \in \mathcal{E}$ joining $v_{i}$ and $F_{j}$ if and only if $v_{i} \in V_{j}$ (i.e., if $v_{i}$ is an argument of $F_{j}$ ).

Let $F(V)$ be a pmf. Then, if the FG corresponding to the factorization of $F$ has no cycles, ${ }^{1}$ the marginal pmfs can be computed exactly in a finite number of steps by the SPA [9]. The SPA is defined by the computation rules at variable and at factor nodes, and by a suitable node activation schedule. Denoting by $\mu_{v_{i} \rightarrow F_{j}}\left(v_{i}\right)$ a message sent from the variable node $v_{i}$ to the factor node $F_{j}$, by $\mu_{F_{j} \rightarrow v_{i}}\left(v_{i}\right)$ a message in the opposite direction, and by $\mathcal{A}_{i}$ the set of functions $F_{j}$ having $v_{i}$ as argument, the message computations performed at variable and factor nodes are, respectively [9]

$$
\begin{aligned}
& \mu_{v_{i} \rightarrow F_{j}}\left(v_{i}\right)=\prod_{H \in \mathcal{A}_{i} \backslash\left\{F_{j}\right\}} \mu_{H \rightarrow v_{i}}\left(v_{i}\right) \\
& \mu_{F_{j} \rightarrow v_{i}}\left(v_{i}\right)=\sum_{\sim\left\{v_{i}\right\}}\left[F_{j}\left(\left\{w \in V_{j}\right\}\right) \prod_{w \in V_{j} \backslash\left\{v_{i}\right\}} \mu_{w \rightarrow F_{j}}(w)\right]
\end{aligned}
$$

where, following the notation of [9], we indicate by $\sum_{\sim\left\{v_{i}\right\}}$ the summary operator, i.e., a sum over all variables excluding $v_{i}$. In the absence of cycles in the graph, the computation usually starts at the leaves of the graph and proceeds until two messages have been passed over every edge, one in each direction.

\section{B. MAP Sequence Detection: Ungerboeck and Forney Ap- proaches}

The Ungerboeck approach for MAP sequence detection [1] is based on the samples $\left\{x_{k}\right\}$ at the output of a filter matched to the received pulse $p(t)$. The sample at time $k T$, can be

\footnotetext{
${ }^{1} \mathrm{~A}$ cycle is a closed path in the graph.
} 
expressed as

$$
x_{k}=\sum_{\ell=-L}^{L} g_{\ell} a_{k-\ell}+n_{k}
$$

where $L$ is a suitable integer parameter, $\mathbf{a}=\left\{a_{k}\right\}$ is the sequence of $M$-ary transmitted symbols, assumed independent, it is

$$
g_{\ell}=\left.p(t) \otimes p^{*}(-t)\right|_{t=\ell T}
$$

and the noise samples $\left\{n_{k}\right\}$ have autocorrelation $R_{n}(m)=$ $2 N_{0} g_{m}$, having denoted by $N_{0}$ the one-sided noise power spectral density. By representing the received signal onto an orthonormal basis and denoting by $\mathbf{r}$ its vector representation, as shown in [1], it is possible to express ${ }^{2}$

$$
p(\mathbf{r} \mid \mathbf{a}) \propto \prod_{k} G_{k}\left(a_{k}, a_{k-1}, \ldots, a_{k-L}\right)
$$

where

$$
\begin{aligned}
& G_{k}\left(a_{k}, a_{k-1}, \ldots, a_{k-L}\right) \\
& =\exp \left\{\frac{1}{N_{0}} \operatorname{Re}\left[x_{k} a_{k}^{*}-\frac{1}{2}\left|a_{k}\right|^{2} g_{0}-\sum_{\ell=1}^{L} a_{k}^{*} a_{k-\ell} g_{\ell}\right]\right\} .
\end{aligned}
$$

For notational convenience, we omitted the dependence of $G_{k}$ on $x_{k}$. Notice that the functions $G_{k}\left(a_{k}, a_{k-1}, \ldots, a_{k-L}\right)$ are not pdfs (nor proportional to pdfs). The MAP sequence detection, based on the following decision rule

$$
\begin{aligned}
\hat{\mathbf{a}}= & \underset{\mathbf{a}}{\arg \max _{\mathbf{a}}} P(\mathbf{a} \mid \mathbf{r}) \\
= & \underset{\mathbf{a}}{\arg \max _{\mathbf{a}}} p(\mathbf{r} \mid \mathbf{a}) P(\mathbf{a})=\underset{\mathbf{a}}{\operatorname{argmax}}[\ln p(\mathbf{r} \mid \mathbf{a})+\ln P(\mathbf{a})] \\
= & \underset{\mathbf{a} g \max _{\mathbf{a}}}{\arg }\left[\ln G_{k}\left(a_{k}, a_{k-1}, \ldots, a_{k-L}\right)+\ln P\left(a_{k}\right)\right] \\
= & \underset{\mathbf{a}}{\operatorname{argmax}} \sum_{k} \operatorname{Re}\left[x_{k} a_{k}^{*}-\frac{1}{2}\left|a_{k}\right|^{2} g_{0}-\sum_{\ell=1}^{L} a_{k}^{*} a_{k-\ell} g_{\ell}\right] \\
& +N_{0} \ln P\left(a_{k}\right)
\end{aligned}
$$

can be implemented by using the VA working on a trellis whose state is defined as $\sigma_{k}=\left(a_{k-1}, \ldots, a_{k-L}\right)$ and by using the branch metrics

$$
\begin{aligned}
\lambda_{k}\left(a_{k}, \sigma_{k}\right)= & \operatorname{Re}\left[x_{k} a_{k}^{*}-\frac{1}{2}\left|a_{k}\right|^{2} g_{0}-\sum_{\ell=1}^{L} a_{k}^{*} a_{k-\ell} g_{\ell}\right] \\
& +N_{0} \ln P\left(a_{k}\right) .
\end{aligned}
$$

The number of trellis states is $S=M^{L}$. Hence, the integer parameter $L$ represents the channel memory.

The Forney approach [2] is based on the output $\left\{y_{k}\right\}$ of a WMF which can be expressed as

$$
y_{k}=\sum_{\ell=0}^{L} f_{\ell} a_{k-\ell}+w_{k}
$$

\footnotetext{
${ }^{2}$ In this letter, we use extensively the proportionality relationship $f \propto g$, indicating that $f=a g$ for some real constant $a$. In fact, a real constant is irrelevant in the maximization to be performed to implement MAP sequence detection whereas on the other hand, the SPA is defined up to scaling its messages by positive factors, independent of the variables represented in the graph.
}

where $\left\{f_{\ell}\right\}_{\ell=0}^{L}$ is the equivalent discrete-time white noise channel impulse response ${ }^{3}$ and now the noise samples $\left\{w_{k}\right\}$ represent a discrete-time white Gaussian noise process. Hence, it is [2]

$$
p(\mathbf{y} \mid \mathbf{a})=\prod_{k} p\left(y_{k} \mid \mathbf{a}\right)
$$

where

$p\left(y_{k} \mid \mathbf{a}\right)=p\left(y_{k} \mid a_{k}, \sigma_{k}\right) \propto \exp \left\{-\left.\frac{1}{2 N_{0}}\left|y_{k}-\sum_{\ell=0}^{L} f_{\ell} a_{k-\ell}\right|\right|_{(10)} ^{2}\right\}$.

Since the sequence $\left\{y_{k}\right\}$ represents a sufficient statistic [2], the MAP sequence detection strategy can be equivalently expressed as

$$
\begin{aligned}
\hat{\mathbf{a}} & =\underset{\mathbf{a}}{\operatorname{argmax}} P(\mathbf{a} \mid \mathbf{y}) \\
& =\underset{\mathbf{a}}{\operatorname{argmax}} p(\mathbf{y} \mid \mathbf{a}) P(\mathbf{a})=\underset{\mathbf{a}}{\operatorname{argmax}}[\ln p(\mathbf{y} \mid \mathbf{a})+\ln P(\mathbf{a})] \\
& =\underset{\mathbf{a}}{\operatorname{argmax}} \sum_{k}\left\{\left|y_{k}-\sum_{\ell=0}^{L} f_{\ell} a_{k-\ell}\right|^{2}+2 N_{0} \ln P\left(a_{k}\right)\right\}
\end{aligned}
$$

and can be implemented by means of the VA working on the same trellis previously defined, with branch metrics

$$
\lambda_{k}\left(a_{k}, \sigma_{k}\right)=\left|y_{k}-\sum_{\ell=0}^{L} f_{\ell} a_{k-\ell}\right|^{2}+2 N_{0} \ln P\left(a_{k}\right) .
$$

These two approaches for MAP sequence detection are exactly equivalent in terms of performance (since they are two alternative formulation of the same strategy and no approximations are involved), and trellis complexity. The only difference is in the expression of the VA branch metrics. For an in deep analysis of the computational advantages of the branch metrics (7), see the relevant discussion in [1]. In addition, the Ungerboeck approach does not require the implementation of the whitening filter necessary to obtain the sufficient statistics (8).

\section{MAP SyMBOL DETECTION BASED ON THE UNGERBOECK APPROACH}

The MAP symbol detection strategy is based on the maximization of the a posteriori probabilities (APPs) of the transmitted symbols given the received signal (or a sufficient statistics):

$$
\hat{a}_{k}=\underset{a_{k}}{\operatorname{argmax}} P\left(a_{k} \mid \mathbf{r}\right)=\underset{a_{k}}{\operatorname{argmax}} P\left(a_{k} \mid \mathbf{y}\right) .
$$

All the well-known materialization of this strategy (e.g., see [6]-[8]) are based on the Forney observation model. In fact, they exploit the property that the sample $y_{k}$ depends on symbols $a_{k}, a_{k-1}, \ldots, a_{k-L}$ only and that Gaussian noise is white. This property does not hold for samples $x_{k}$ and, in fact, as previously mentioned, the factorization (4) does not involve pdfs. Hence, the probabilistic derivation usually employed to derive MAP symbol detection algorithms [6]-[8] cannot be extended to the Ungerboeck observation model.

\footnotetext{
${ }^{3}$ The coefficients $\left\{f_{\ell}\right\}$ can be derived from coefficients $\left\{g_{\ell}\right\}$ by means of a spectral factorization procedure.
} 


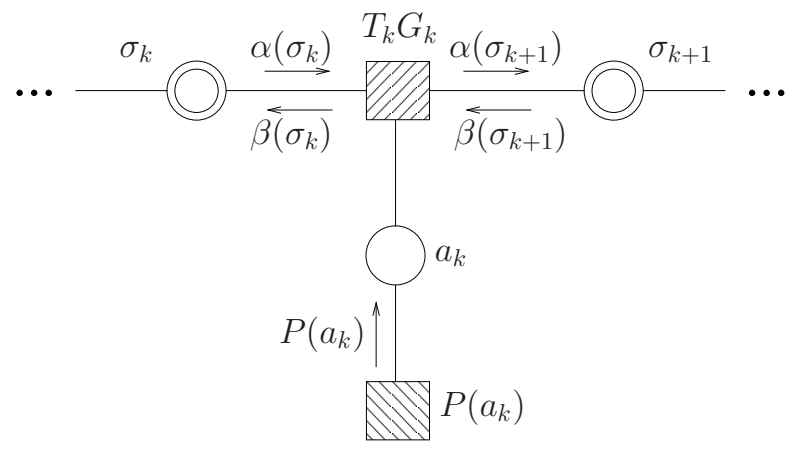

Fig. 1. Factor graph corresponding to eqn. (14).

A MAP symbol detection algorithm for the Ungerboeck observation model can, however, be obtained by using the FG/SPA framework. We construct the FG corresponding to the joint APP of the transmitted symbols and the trellis states given the received signal (the same used for MAP sequence detection) and let the SPA compute the marginal APPs $P\left(a_{k} \mid \mathbf{r}\right)$ necessary to implement MAP symbol detection. To obtain this result, we use the property that a FG represents the factorization of a pmf as the product of local functions that are not necessarily pmfs or pdfs [9].

The joint APP of the transmitted symbols and the trellis states $\boldsymbol{\sigma}=\left\{\sigma_{k}\right\}$ given the received signal can be expressed as

$$
\begin{aligned}
P(\mathbf{a}, \boldsymbol{\sigma} \mid \mathbf{r}) & \propto p(\mathbf{r} \mid \mathbf{a}) P(\boldsymbol{\sigma} \mid \mathbf{a}) P(\mathbf{a}) \\
& =\prod_{k} G_{k}\left(a_{k}, \sigma_{k}\right) T_{k}\left(a_{k}, \sigma_{k}, \sigma_{k+1}\right) P\left(a_{k}\right)
\end{aligned}
$$

where the function $T_{k}\left(a_{k}, \sigma_{k}, \sigma_{k+1}\right)$ is the trellis indicator function equal to 1 if $a_{k}, \sigma_{k}$, and $\sigma_{k+1}$ satisfy the trellis definition and to zero otherwise [9]. In fact, since a and $\sigma$ are in a one-to-one correspondence, the probability $P(\boldsymbol{\sigma} \mid \mathbf{a})$ is equal to 1 if all the trellis constraints are verified and to zero otherwise.

The Wiberg-type graph [9], [10] corresponding to the joint pmf in (14) is sketched in Fig. 1, where the hidden variables $\left\{\sigma_{k}\right\}$ have been explicitly represented. With reference to the messages in the figure, by applying the updating rules of the SPA, messages $\alpha\left(\sigma_{k}\right)$ and $\beta\left(\sigma_{k}\right)$ can be recursively computed by means of the following forward and backward recursions:

$$
\begin{aligned}
\alpha\left(\sigma_{k+1}\right) & =\sum_{\sim\left\{\sigma_{k+1}\right\}} \alpha\left(\sigma_{k}\right) T_{k}\left(a_{k}, \sigma_{k}, \sigma_{k+1}\right) G_{k}\left(a_{k}, \sigma_{k}\right) P\left(a_{k}\right) \\
\beta\left(\sigma_{k}\right) & =\sum_{\sim\left\{\sigma_{k}\right\}} \beta\left(\sigma_{k+1}\right) T_{k}\left(a_{k}, \sigma_{k}, \sigma_{k+1}\right) G_{k}\left(a_{k}, \sigma_{k}\right) P\left(a_{k}\right)
\end{aligned}
$$

whose initial conditions can be easily derived. Finally, the marginal symbol APPs can be computed by means of the following completion

$$
\begin{aligned}
& P\left(a_{k} \mid \mathbf{r}\right)= \\
& \quad P\left(a_{k}\right) \sum_{\sim\left\{a_{k}\right\}} \alpha\left(\sigma_{k}\right) \beta\left(\sigma_{k+1}\right) T_{k}\left(a_{k}, \sigma_{k}, \sigma_{k+1}\right) G_{k}\left(a_{k}, \sigma_{k}\right) .
\end{aligned}
$$

Since the employed FG is cycle-free, the marginal APPs computed by the SPA are exact. Therefore, the derived algorithm is an alternative optimal implementation of the MAP symbol detection strategy. As such, the performance is the same that can be obtained, for example, by using the BCJR algorithm based on the Forney model, and for this reason, no simulation results are reported here. The structure of the algorithm is also the same. In fact, we have a forward and a backward recursion and a final completion, all working on the same trellis of the original BCJR algorithm. The only difference is that the term $G_{k}\left(a_{k}, \sigma_{k}\right)$ in (15), (16), and (17), is replaced, in the classical BCJR algorithm, by $p\left(y_{k} \mid a_{k}, \sigma_{k}\right)$ given in (10) [6], [9]. Hence the difference is in the expression of the branch metrics only, as for MAP sequence detection. As a consequence, for a complexity comparison between the proposed algorithm and the classical BCJR algorithm based on the Forney model, the same considerations mentioned in the previous section still hold.

\section{CONCLUSions}

In this letter, a forward-backward algorithm which implements optimal MAP symbol detection has been derived for ISI channels and the Ungerboeck observation model. The algorithm is similar in structure to the well-known BCJR algorithm. As for MAP sequence detection, the Ungerboeck and Forney observation models are exactly equivalent for MAP symbol detection also.

\section{REFERENCES}

[1] G. Ungerboeck, "Adaptive maximum likelihood receiver for carriermodulated data-transmission systems," IEEE Trans. Commun., vol. 22, pp. 624-636, May 1974.

[2] G. D. Forney, Jr., "Lower bounds on error probability in the presence of large intersymbol interference," IEEE Trans. Commun., vol. 20, pp. 76-77, Feb. 1972.

[3] C. Douillard, M. Jezequel, C. Berrou, A. Picart, P. Didier, and A. Glavieux, "Iterative correction of intersymbol interference: turboequalization," European Trans. Telecommun., vol. 6, pp. 507-511, Sept./Oct. 1995.

[4] A. Picart, P. Didier, and A. Glavieux, "Turbo-detection: A new approach to combat channel frequency selectivity," in Proc. IEEE International Conf. Commun., June 1997.

[5] M. Tüchler, R. Koetter, and A. C. Singer, "Turbo equalization: Principles and new results," IEEE Trans. Commun., vol. 55, pp. 754-767, May 2002.

[6] L. R. Bahl, J. Cocke, F. Jelinek, and J. Raviv, "Optimal decoding of linear codes for minimizing symbol error rate," IEEE Trans. Inform. Theory, vol. 20, pp. 284-287, Mar. 1974.

[7] K. Abend and B. D. Fritchman, "Statistical detection for communication channels with intersymbol interference," in Proc. IEEE, vol. 58, pp. 779-785, May 1970.

[8] Y. Li, B. Vucetic, and Y. Sato, "Optimum soft-output detection for channels with intersymbol interference," IEEE Trans. Inform. Theory, vol. 41, pp. 704-713, May 1995.

[9] F. R. Kschischang, B. J. Frey, and H.-A. Loeliger, "Factor graphs and the sum-product algorithm," IEEE Trans. Inform. Theory, vol. 47, pp. 498-519, Feb. 2001.

[10] N. Wiberg, "Codes and decoding on general graphs," Ph.D. dissertation, Linköping University (Sweden), 1996. 